\title{
SIMULTANEOUS OBSERVATIONS OF COMET C/2002 T7 (LINEAR) WITH THE BERKELEY-ILLINOIS-MARYLAND ASSOCIATION AND OWENS VALLEY RADIO OBSERVATORY INTERFEROMETERS: $\mathrm{HCN}$ AND $\mathrm{CH}_{3} \mathrm{OH}$
}

\author{
Michiel R. Hogerheijde ${ }^{1}$, Chunhua QI $^{2}$, Imke de Pater ${ }^{3,9}$, Geoffrey A. Blake ${ }^{4}, 10$, D. N. Friedel ${ }^{5}$, J. R. Forster ${ }^{6}$, \\ Patrick Palmer $^{7}$, Anthony J. Remijan ${ }^{8}$, L. E. SNyder ${ }^{5}$, And M. C. H. Wright ${ }^{3}$ \\ ${ }^{1}$ Leiden Observatory, Leiden University, P.O. Box 9513, 2300 RA, Leiden, Netherlands; michiel@ strw.leidenuniv.nl \\ ${ }^{2}$ Harvard-Smithsonian Center for Astrophysics, 60 Garden Street, Cambridge, MA 02138, USA \\ ${ }^{3}$ Department of Astronomy, 601 Campbell Hall, University of California at Berkeley, Berkeley, CA 94720, USA \\ ${ }^{4}$ Division of Geophysics and Planetary Science, MS 150-21, California Institute of Technology, 1200 East California Boulevard, Pasadena, CA 91125, USA \\ ${ }^{5}$ Department of Astronomy, University of Illinois, 1002 West Green Street, Urbana, IL 61801, USA \\ ${ }^{6}$ Hat Creek Radio Observatory, 42231 Bidwell Road, Hat Creek, CA 96040, USA \\ ${ }^{7}$ Department of Astronomy and Astrophysics, University of Chicago, 5640 South Ellis Avenue, Chicago, IL 60637, USA \\ ${ }^{8}$ National Radio Astronomy Observatory, 520 Edgemont Rd, Charlottesville, VA 22901, USA \\ Received 2008 May 28; accepted 2009 March 25; published 2009 April 29
}

\begin{abstract}
We present observations of $\mathrm{HCN} J=1-0$ and $\mathrm{CH}_{3} \mathrm{OH} J\left(K_{a}, K_{c}\right)=3(1,3)-4(0,4) \mathrm{A}^{+}$emission from comet C/2002 T7 (LINEAR) obtained simultaneously with the Owens Valley Radio Observatory (OVRO) and BerkeleyIllinois-Maryland Association (BIMA) millimeter interferometers. We combined the data from both arrays to increase the $(u, v)$ sampling and signal to noise of the detected line emission. We also report the detection of $\mathrm{CH}_{3} \mathrm{OH} J\left(K_{a}, K_{c}\right)=8(0,8)-7(1,7) \mathrm{A}^{+}$with OVRO data alone. Using a molecular excitation code that includes the effects of collisions with water and electrons, as well as pumping by the Solar infrared photons (for HCN alone), we find a production rate of $\mathrm{HCN}$ of $2.9 \times 10^{26} \mathrm{~s}^{-1}$ and for $\mathrm{CH}_{3} \mathrm{OH}$ of $2.2 \times 10^{27} \mathrm{~s}^{-1}$. Compared to the adopted water production rate of $3 \times 10^{29} \mathrm{~s}^{-1}$, this corresponds to an $\mathrm{HCN} / \mathrm{H}_{2} \mathrm{O}$ ratio of $0.1 \%$ and a $\mathrm{CH}_{3} \mathrm{OH} / \mathrm{H}_{2} \mathrm{O}$ ratio of $0.7 \%$. We critically assess the uncertainty of these values due to the noise $(\sim 10 \%)$, the uncertainties in the adopted comet model ( $\sim 50 \%$ ), and the uncertainties in the adopted collisional excitation rates (up to a factor of 2). Pumping by Solar infrared photons is found to be a minor effect for HCN, because our $15^{\prime \prime}$ synthesized beam is dominated by the region in the coma where collisions dominate. Since the uncertainties in the derived production rates are at least as large as one-third of the differences found between comets, we conclude that reliable collision rates and an accurate comet model are essential. Because the collisionally dominated region critically depends on the water production rate, using the same approximate method for different comets may introduce biases in the derived production rates. Multiline observations that directly constrain the molecular excitation provide much more reliable production rates.
\end{abstract}

Key words: comets: individual (LINEAR C/2002 T7) - molecular processes - radio lines: solar system techniques: interferometric

\section{INTRODUCTION}

Imaging radio emissions from comets has been notoriously difficult because of the rather weak signals and relatively large apparent scales, the combination of which leads to low signal-tonoise images. Since it is important to image comets at all relevant scales (arcseconds-degrees) to extract the detailed emission (excitation) and outflow properties, we have used in the past both single dishes (or phased arrays) and interferometers in tandem to investigate the detailed emission characteristics of some cometary species (e.g., Wright et al. 1998; Hogerheijde et al. 2004; Milam et al. 2006). Based upon such combined observations of the extremely bright comet C/1995 O1 (HaleBopp), Wright et al. (1998) showed that the HCN emission is usually confined to the "near" nucleus region (radius $\lesssim 1^{\prime}$, or $70,000 \mathrm{~km}$ at the moment of observations, at a heliocentric distance of $\sim 1 \mathrm{AU}$ ), so that interferometric observations at millimeter wavelengths carried out with antennas a few meters in size (such as the Berkeley-Illinois-Maryland Association (BIMA) array, with $6 \mathrm{~m}$ dishes) will indeed sample all the

\footnotetext{
9 Faculty of Aerospace Engineering, Delft University of Technology, P.O. Box 5058, 2600 GB, Delft, Netherlands.

${ }^{10}$ Division of Chemistry and Chemical Engineering, California Institute of Technology, 1200 East California Boulevard, Pasadena, CA 91125, USA.
}

cometary emissions of this species. In contrast, $\mathrm{HCO}^{+}$emissions extend over an area many arcminutes in size $(300,000 \mathrm{~km}$; Womack et al. 1997; Lovell et al. 1998; Wright et al. 1998), and such large-scale emissions are largely lost in interferometric images due to the "missing short spacing" problem. A proper excitation and radiative transfer calculation can in principle take the filtering properties of an interferometer into account exactly, loosening the constraints on the observational setup.

The volatile composition of comets is comprehensively reviewed by Bockelée-Morvan et al. (2004), based in part on the comparative study of Biver et al. (2002) (see also Biver et al. 2006). These authors conclude that $\mathrm{HCN}$ has a remarkably constant abundance with respect to water of $\mathrm{HCN} / \mathrm{H}_{2} \mathrm{O} \approx$ $0.1 \%$ and can be used effectively as a proxy for water. Other species, for example methanol, show much larger variations from comet to comet and Bockelée-Morvan et al. (2004) summarize $\mathrm{CH}_{3} \mathrm{OH} / \mathrm{H}_{2} \mathrm{O}$ ratios ranging from $<0.15 \%$ to $6 \%$, with many comets around $2 \%$. The studied sample size is too small to draw any conclusions on the correlation between abundances and the different dynamic families of comets. More observations are needed to establish any such correlation, and to ultimately asses the link between cometary composition and the formation and evolution of the solar system (Bockelée-Morvan et al. 2004). 
Table 1

Summary of Observations

\begin{tabular}{lcrrr}
\hline \hline Date & Transition & $\begin{array}{c}\text { Frequency } \\
(\mathrm{GHz})\end{array}$ & $\begin{array}{c}r_{h}{ }^{\mathrm{a}} \\
(\mathrm{AU})\end{array}$ & $\begin{array}{c}\Delta^{\mathrm{b}} \\
(\mathrm{AU})\end{array}$ \\
\hline 2004 May 11.70-11.87 & $\mathrm{HCN} J=1-0$ & $88.63185^{\mathrm{c}}$ & 0.73 & 0.44 \\
2004 May 11.60-11.87 & $\mathrm{CH}_{3} \mathrm{OH} J\left(K_{a}, K_{c}\right)=8(0,8)-7(1,7) \mathrm{A}^{+\mathrm{d}}$ & 95.16944 & 0.73 & 0.44 \\
2004 May 13.70-13.87 & $\mathrm{CH}_{3} \mathrm{OH} J\left(K_{a}, K_{c}\right)=3(1,3)-4(0,4) \mathrm{A}^{+}$ & 107.01385 & 0.75 & 0.38
\end{tabular}

Notes.

${ }^{a}$ Heliocentric distance.

${ }^{\mathrm{b}}$ Geocentric distance.

${ }^{c}$ Adopted frequency for receiver tuning. The $\mathrm{HCN} J=1-0$ hyperfine lines are $F=1-1$ at $88.63041 \mathrm{GHz}$ (at a relative velocity $\Delta v$ of $+4.7 \mathrm{~km} \mathrm{~s}^{-1}$ with respect to the tuning frequency, $F=2-1$ at $88.63185 \mathrm{GHz}$ (at $\Delta v=$ $0 \mathrm{~km} \mathrm{~s}^{-1}$ ), and $F=0-1$ at $88.63394 \mathrm{GHz}$ (at $\Delta v=-7.1 \mathrm{~km} \mathrm{~s}^{-1}$.

${ }^{\mathrm{d}} \mathrm{CH}_{3} \mathrm{OH} J\left(K_{a}, K_{c}\right)=8(0,8)-7(1,7) \mathrm{A}^{+}$was only observed with OVRO.

In the spring of 2004 comet $\mathrm{C} / 2002 \mathrm{~T} 7$ (LINEAR), hereafter comet LINEAR, passed through the inner solar system, and came to within $0.3 \mathrm{AU}$ from the Earth. The comet was observed with BIMA ${ }^{11}$ and the Owens Valley Radio Observatory $\left(\mathrm{OVRO}^{12}\right)$ in 2004 May, just before the two arrays were dismantled and moved to the Inyo mountains in southern California, where they now operate as a single interferometer, the Combined Association for Research in Millimeter-wave Astronomy $\left(\right.$ CARMA $\left.^{13}\right)$.

An overview of all our BIMA observations for comet LINEAR, including a list of all detected species as well as upper limits for all species searched for but not detected, was given by Remijan et al. (2006). BIMA detections of HCN and derived production rates were published by Friedel et al. (2005). In a subsequent paper, Remijan et al. (2008) compare BIMA array data with single dish measurements obtained with the $12 \mathrm{~m}$ Arizona Radio Observatory (ARO). The authors derived an excitation temperature of $35 \pm 5 \mathrm{~K}$ and production rate of $2.0 \pm 0.1 \times 10^{27} \mathrm{~s}^{-1}$ for methanol in comet LINEAR, and concluded that methanol is a parent nuclear species, i.e., the molecules sublime directly from the nucleus. In this paper, we present the successful $\mathrm{HCN} J=1-0$ and $\mathrm{CH}_{3} \mathrm{OH}$ $J\left(K_{a}, K_{c}\right)=3(1,3)-4(0,4) \mathrm{A}^{+}$and $8(0,8)-7(1,7) \mathrm{A}^{+}$observations of comet LINEAR that were obtained with the "virtual CARMA" (vCARMA) array, i.e., observations obtained simultaneously with the BIMA and OVRO arrays. We analyze these data with a molecular excitation model based on the radiative transfer code from Hogerheijde \& van der Tak (2000), which was adapted for the excitation conditions in cometary comae following the work of Bensch \& Bergin (2004). Section 2 lists the observational details. Section 3 presents the basic results, which are further discussed in Section 4, where the above-mentioned model is used to derive production rates and to test the distribution and source of the detected molecules. Section 5 summarizes our main conclusions and describes the future potential of our computational method with arrays such as CARMA and ultimately the Atacama Large Millimeter Array (ALMA).

\footnotetext{
11 BIMA was operated by the University of California, Berkeley, the University of Illinois, and the University of Maryland with support from the National Science Foundation.

12 OVRO was operated by the California Institute of Technology with support from the National Science Foundation.

13 Support for CARMA construction was derived from the states of California, Illinois, and Maryland, the Gordon and Betty Moore Foundation, the Kenneth T. and Eileen L. Norris Foundation, the Associates of the California Institute of Technology, and the National Science Foundation. Ongoing CARMA development and operations are supported by the National Science Foundation under a cooperative agreement, and by the CARMA partner universities.
}

\section{OBSERVATIONS}

We observed comet C/2002 T7 (LINEAR) simultaneously with BIMA at the Hat Creek Radio Observatory in California $\left(121^{\circ} 28^{\prime} 18^{\prime \prime} .5\right.$ West, $40^{\circ} 49^{\prime} 2^{\prime \prime} .5$ North, altitude $\left.1043 \mathrm{~m}\right)$ and OVRO at the Owens Valley Radio Observatory near Big Pine, California $\left(118^{\circ} 16^{\prime} 56^{\prime \prime}\right.$ West, $37^{\circ} 14^{\prime} 02^{\prime \prime}$ North, altitude $\left.1220 \mathrm{~m}\right)$ in the period 2004 May 11-15. Emission from HCN $J=1-0$ and $\mathrm{CH}_{3} \mathrm{OH} J\left(K_{a}, K_{c}\right)=3(1,3)-4(0,4) \mathrm{A}^{+}$were recorded with both arrays on 2004 May 11 and 13, respectively. On May $11(\mathrm{HCN})$, the comet was at a heliocentric distance $r=0.73 \mathrm{AU}$ and a geocentric distance $\Delta=0.44 \mathrm{AU}$; on May $13\left(\mathrm{CH}_{3} \mathrm{OH}\right)$, $r=0.75 \mathrm{AU}, \Delta=0.38$. At $\Delta=0.44 \mathrm{AU}$ and $0.38 \mathrm{AU}$, respectively, $1^{\prime \prime}=319 \mathrm{~km}$ and $276 \mathrm{~km}$, so our $10^{\prime \prime}-20^{\prime \prime}$ beam samples scales of approximately $5000 \mathrm{~km}$. Table 1 summarizes the observations.

During the observations, both arrays were in compact configurations, with the ten $6 \mathrm{~m}$ BIMA array providing projected baselines of $\sim 6-35 \mathrm{~m}$ in length, and the six $10 \mathrm{~m}$ OVRO dishes projected baselines of $\sim 9-45 \mathrm{~m}$. This resulted in naturally weighted synthesized beams of $23^{\prime \prime} \times 18^{\prime \prime}(\mathrm{BIMA}, \mathrm{HCN})$, $14^{\prime \prime} \times 12^{\prime \prime}(\mathrm{OVRO}, \mathrm{HCN}), 19^{\prime \prime} \times 16^{\prime \prime}\left(\mathrm{BIMA}, \mathrm{CH}_{3} \mathrm{OH}\right)$, and $14^{\prime \prime} \times 10^{\prime \prime}$, and $13^{\prime \prime} \times 11^{\prime \prime}\left(\mathrm{OVRO}\right.$, both $\mathrm{CH}_{3} \mathrm{OH}$ lines). For $\mathrm{HCN}$ $J=1-0$, the passband was centered on the $J=1-0$ hyperfine lines near $88.6318 \mathrm{GHz}$, and for $\mathrm{CH}_{3} \mathrm{OH} J\left(K_{a}, K_{c}\right)=3(1,3)$ $4(0,4) \mathrm{A}^{+}$on the line rest frequency of $107.0138 \mathrm{GHz}$. The spectral window at BIMA contained 128 channels spread over $50 \mathrm{MHz}$, yielding a spectral resolution of $0.39 \mathrm{MHz}$ $\left(1.3 \mathrm{~km} \mathrm{~s}^{-1}\right)$; at OVRO the 128 channels of the spectral window covered $8 \mathrm{MHz}(\mathrm{HCN})$ and $16 \mathrm{MHz}\left(\mathrm{CH}_{3} \mathrm{OH}\right)$, yielding spectral resolutions of $0.062 \mathrm{MHz}\left(0.21 \mathrm{~km} \mathrm{~s}^{-1}\right)$ and $0.125 \mathrm{MHz}$ $\left(0.35 \mathrm{~km} \mathrm{~s}^{-1}\right)$, respectively. The OVRO data of $\mathrm{CH}_{3} \mathrm{OH}$ $J\left(K_{a}, K_{c}\right)=8(0,8)-7(1,7) \mathrm{A}^{+}$were tuned to $95.16955 \mathrm{GHz}$ with the 128 channels of the spectral bands covering $8 \mathrm{MHz}$ with a resolution of $0.062 \mathrm{MHz}\left(0.20 \mathrm{~km} \mathrm{~s}^{-1}\right)$.

We used JPL Reference orbit $69^{14}$ for the observations at both telescopes to ensure to track the same position on the sky. The positional accuracy of this ephemeris is good to within a few arcseconds, i.e., well within the size of our synthesized beam. Unfortunately, when the ephemeris was coded at OVRO, the geocentric and topocentric coordinate systems were incorrectly switched. This resulted in an apparent daily shift of the comet's position of up to $25^{\prime \prime}$. The amplitude of this shift is much less than the primary beam size of $\sim 80^{\prime \prime}$ and therefore does not affect the individual integrations of 3 minutes long. Before they can

\footnotetext{
14 http://ssd.jpl.nasa.gov/horizons.html
} 
Table 2

Line Intensities

\begin{tabular}{lccc}
\hline \hline Transition & Telescope & $\begin{array}{c}I_{0} \\
\left(\mathrm{Jy} \mathrm{bm}^{-1} \mathrm{~km} \mathrm{~s}^{-1}\right)\end{array}$ & $\begin{array}{c}\text { Beam } \\
\left({ }^{\prime \prime}\right)\end{array}$ \\
\hline $\mathrm{HCN} \mathrm{1-0}$ & BIMA & $0.49 \pm 0.11^{\mathrm{a}}$ & $23 \times 18$ \\
$\mathrm{HCN} \mathrm{1-0}$ & OVRO & $0.30 \pm 0.06^{\mathrm{a}}$ & $14 \times 12$ \\
$\mathrm{HCN} \mathrm{1-0}$ & vCARMA & $0.42 \pm 0.05^{\mathrm{a}}$ & $15 \times 14$ \\
$\mathrm{CH}_{3} \mathrm{OH} 3(1,3)-4(0,4) \mathrm{A}^{+}$ & BIMA & $0.49 \pm 0.11$ & $19 \times 16$ \\
$\mathrm{CH}_{3} \mathrm{OH} 3(1,3)-4(0,4) \mathrm{A}^{+}$ & OVRO & $0.44 \pm 0.10$ & $14 \times 10$ \\
$\mathrm{CH}_{3} \mathrm{OH} 3(1,3)-4(0,4) \mathrm{A}^{+}$ & vCARMA & $0.44 \pm 0.07$ & $14 \times 12$ \\
$\mathrm{CH}_{3} \mathrm{OH} 8(0,8)-7(1,7) \mathrm{A}^{+}$ & OVRO & $0.40 \pm 0.06$ & $13 \times 11$ \\
\hline
\end{tabular}

Note.

${ }^{a}$ Integrated intensity for the main $\mathrm{HCN} J=1-0$ hyperfine component $F=$ 2-1 only.

be combined and formed into an image, each individual 3 min integration was corrected for the positional offset and referenced to the correct phase tracking center. After this procedure, the OVRO data showed cometary emission consistent with the detections from the BIMA observations.

The complex antenna gains of each array were calibrated separately, using the quasar $\mathrm{J} 0108+015$ as a phase calibrator and the source $\mathrm{W} 3(\mathrm{OH})$ as a flux calibrator at BIMA, and the quasar J0116-116 as a phase calibrator and the planet Uranus as a flux calibrator at OVRO. At OVRO, a flux for J0116-116 of $1.0 \mathrm{Jy}$ was found, consistent with contemporaneous measurements at the SubMillimeter Array (SMA); for the quasars 3C84 and 3C454.3, which were also contained in the OVRO track, fluxes of $4.7 \mathrm{Jy}$ and $4.4 \mathrm{Jy}$, respectively, were measured that also agree with values reported at the SMA. At BIMA a flux for J0108+015 of $1.3 \mathrm{Jy}$ was measured, higher by a factor of 1.5 than the $0.89 \mathrm{Jy}$ reported for the same date by the Australia Telescope Compact Array (ATCA). Since the measured $\mathrm{HCN} J=1-0$ and $\mathrm{CH}_{3} \mathrm{OH}$ $J\left(K_{a}, K_{c}\right)=3(1,3)-4(0,4) \mathrm{A}^{+}$line fluxes (see below) at BIMA also exceeded those measured at OVRO by a factor of 1.5 , we scaled the BIMA fluxes down by this same factor (Table 2 only contains these corrected values).

The OVRO data were calibrated using the MMA package specific for OVRO data (Scoville et al. 1993), and the BIMA were calibrated using MIRIAD (Sault et al. 1995). The OVRO data were then converted to the MIRIAD format, and combined with the BIMA data after both data sets were regridded to a common velocity sampling of $0.5 \mathrm{~km} \mathrm{~s}^{-1}$ and an effective resolution of $1.3 \mathrm{~km} \mathrm{~s}^{-1}$, producing a data set that covers projected baseline lengths of 6-45 $\mathrm{m}$. This process has been described in detail by Hogerheijde et al. (2004) The spectral resolution of the $\mathrm{CH}_{3} \mathrm{OH} J\left(K_{a}, K_{c}\right)=8(0,8)-7(1,7) \mathrm{A}^{+}$line that was only observed with OVRO was kept at the original $0.2 \mathrm{~km} \mathrm{~s}^{-1}$. Standard image deconvolution was performed, using natural weighting. Table 2 lists noise levels and synthesized beam sizes of $15^{\prime \prime} \times 14^{\prime \prime}$ for HCN $J=1-0$ and $14^{\prime \prime} \times 12^{\prime \prime}$ for the $\mathrm{CH}_{3} \mathrm{OH} J\left(K_{a}, K_{c}\right)=3(1,3)-4(0,4) \mathrm{A}^{+}$line.

\section{RESULTS}

Figures 1 and 2 present the integrated intensity maps (for HCN $J=1-0$ only the main $F=2-1$ hyperfine component is included) and the spectra over the central synthesized beams for the BIMA and OVRO data individually, and for the combined (vCARMA) data sets. Table 2 lists the integrated intensities, line widths, and beam sizes. Figure 3 shows the $\mathrm{CH}_{3} \mathrm{OH}$ $J\left(K_{a}, K_{c}\right)=8(0,8)-7(1,7) \mathrm{A}^{+}$detection from OVRO alone.
All three transitions are clearly detected, and, for $\mathrm{HCN} J=$ $1-0$ and $\mathrm{CH}_{3} \mathrm{OH} J\left(K_{a}, K_{c}\right)=3(1,3)-4(0,4) \mathrm{A}^{+}$, the combined data sets have a spatial resolution that is intermediate between the individual BIMA and OVRO data, and a higher signal to noise than the individual data sets, as expected. For HCN $J=$ 1-0 the combined data clearly show all three hyperfine components, with relative intensities of 1:0.4 $\pm 0.3: 0.7 \pm 0.4(F=2-1$ : $F=0-1: F=1-1)$ which within the noise are consistent with optically thin lines in local thermodynamic equilibrium (LTE; 1:0.2:0.6, JPL Molecular Spectroscopy Database; Pickett et al. 1998). The integrated intensity images show no obvious structure, beyond that expected for a spherically symmetric expanding coma. The line widths are consistent with expansion velocities of order $0.5-1.0 \mathrm{~km} \mathrm{~s}^{-1}$ (compared to Friedel et al. 2005, who find $0.62 \mathrm{~km} \mathrm{~s}^{-1}$ ), with the line centered close to $0 \mathrm{~km} \mathrm{~s}^{-1}$ (cometocentric frame) corresponding to the systemic velocity of the comet as described through the adopted ephemeris. Both $\mathrm{CH}_{3} \mathrm{OH}$ lines appear offset by $\sim 0.5 \mathrm{~km} \mathrm{~s}^{-1}$ while the image in the OVRO observations appears slightly shifted in position (by several arcseconds; less than the synthesized beam), but we cannot find a reason for this and no shifts were noted by Remijan et al. (2006) for the same $\mathrm{CH}_{3} \mathrm{OH}$ line observed with BIMA only. We conclude that the apparent shifts may be due to noise in the data affecting the line profile shape, or due to imperfections in the processing of the OVRO data. Given these uncertainties we do not venture to explain this shift. A detailed comparison between the observations and an expanding, nuclear source for $\mathrm{HCN}$ and $\mathrm{CH}_{3} \mathrm{OH}$ (Haser distribution) is presented in the following section.

\section{DISCUSSION}

The emission in molecular lines is determined by their excitation via collisions with, predominantly, water and electrons, and via absorption of line and continuum photons, including those of the solar (infrared) spectrum (see, for example, Crovisier 1987, and the comprehensive review by Bockelée-Morvan et al. 2004). In the inner regions of the coma, collisions will dominate the excitation, which is expected to be close to thermal equilibrium. In the outer regions of the coma, fluorescent equilibrium will be reached: absorption of a solar infrared photon results in excitation into an excited vibrational level. This is followed almost immediately by spontaneous emission of a photon (in the infrared). Because of the selection rules for these radiative transitions, the molecule can move up or down in rotational energy by up to two $(J)$ levels for each cycle, and the final distribution over $J$ levels will be dictated by the solar flux and the spontaneous radiative decay rates. The location in the coma of the transition from collisional to fluorescent equilibrium depends on the solar flux at the time and on the production rate of water (and electrons) which determines the efficiency of collisional excitation. For comet LINEAR, Friedel et al. (2005) argue that up to radii of $80^{\prime \prime}(26,000 \mathrm{~km})$ from the nucleus collisions dominate excitation, while regions beyond radii of $120^{\prime \prime}(40,000 \mathrm{~km})$ are expected to be in fluorescent equilibrium.

\subsection{Comet Model}

The resulting line strength depends on the abundance and spatial distribution of the molecules. For parent species, such as $\mathrm{H}_{2} \mathrm{O}, \mathrm{HCN}$, and $\mathrm{CH}_{3} \mathrm{OH}$, the density in the coma drops with distance from the nucleus, which we assume to be the sole 

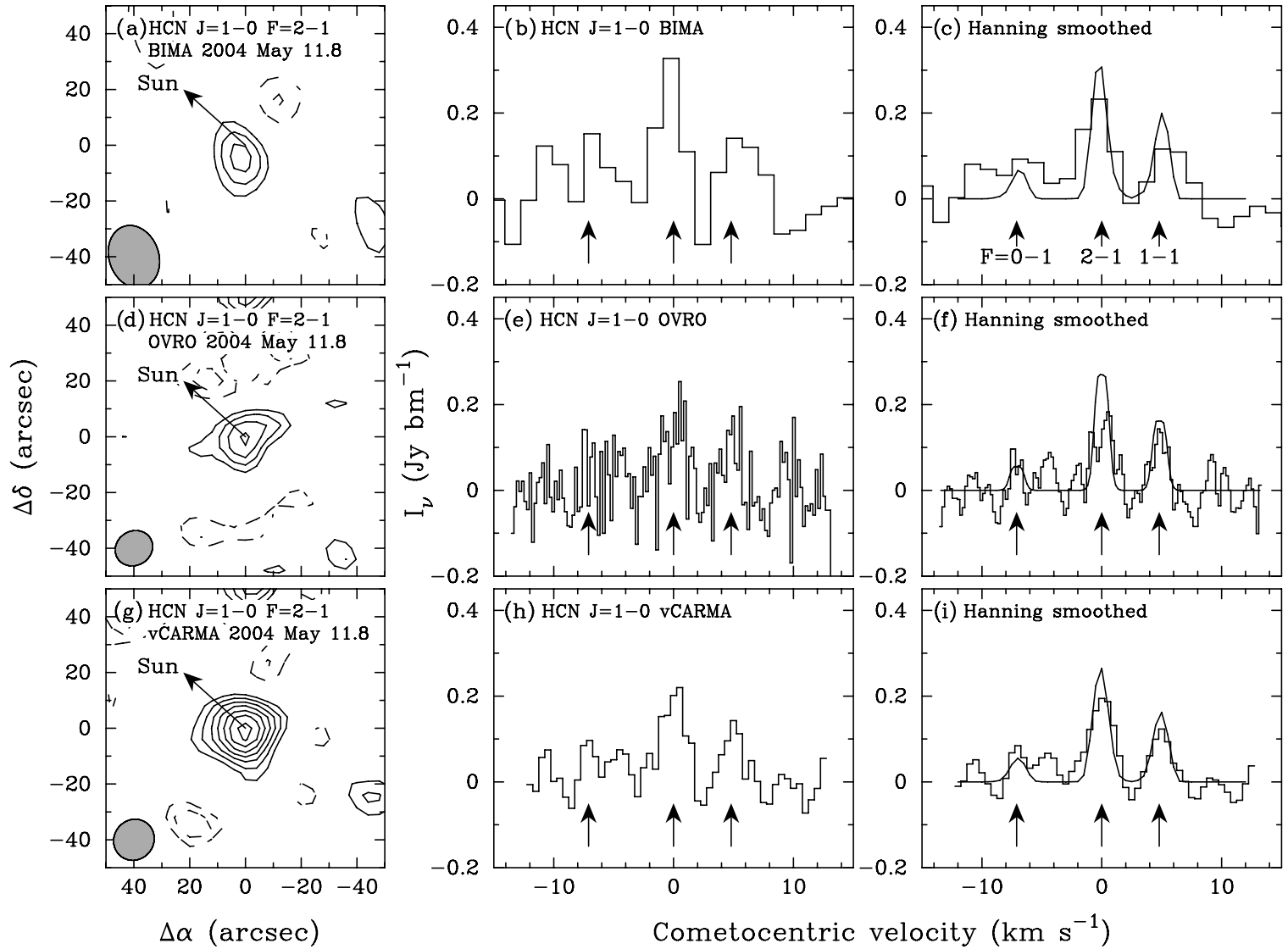

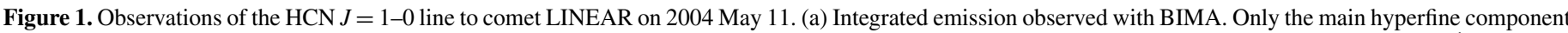

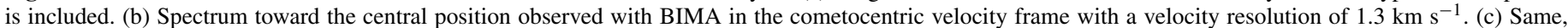

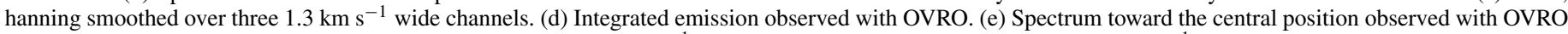

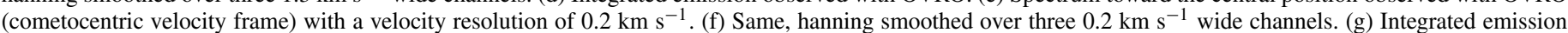

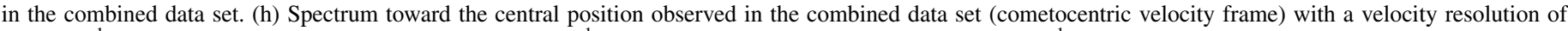

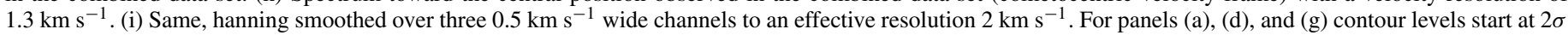

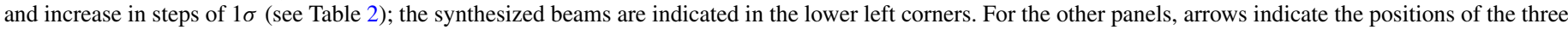
hyperfine components of the HCN transition. The smooth solid line in panels (c), (f), and (i) shows the best-fit model from Section 4.3.

source of $\mathrm{H}_{2} \mathrm{O}, \mathrm{HCN}$, and $\mathrm{CH}_{3} \mathrm{OH}$ molecules. The decrease in gas density can be calculated using the Haser model with a constant outflow velocity, $v_{o}$, (Haser 1957) as

$$
n(r)=\frac{Q}{4 \pi r^{2} v_{o}} \exp ^{-\frac{\left(r-r_{n}\right)}{r_{\lambda}}},
$$

with $n$ is the number density of molecules, $Q$ is the production rate, $r$ is the cometocentric distance, $r_{n}$ is the radius of the nucleus, and $r_{\lambda}$ is the characteristic photodissociation length. We adopt $v_{o}=0.62 \mathrm{~km} \mathrm{~s}^{-1}$ from Friedel et al. (2005). Equation (1) provides the spatial distribution of water and of the species of interest, by adopting the relevant production rates $Q$ and photodissociation lengths $r_{\lambda}$. For water, we adopt a production rate of $Q\left(\mathrm{H}_{2} \mathrm{O}\right)=3.0 \times 10^{29} \mathrm{~s}^{-1}$ which is appropriate for the date of the observations (Biver et al. 2007; DiSanti et al. 2006). The production rates of $\mathrm{HCN}$ and $\mathrm{CH}_{3} \mathrm{OH}$ are free parameters in our model and will be derived in Section 4.3. For water, we adopt a dissociation rate $\beta=1.0 \times$ $10^{-5} \mathrm{~s}^{-1}$ at a heliocentric distance $r_{h}=1$ AU yielding $r_{\lambda}=$ $v_{o} / \beta=62,000 \mathrm{~km}$; for HCN we adopt $\beta=1.5 \times 10^{-5} \mathrm{~s}^{-1}$ at $r_{h}=1 \mathrm{AU}$ so that $r_{\lambda}=41,000 \mathrm{~km}$; for $\mathrm{CH}_{3} \mathrm{OH}$, we adopt a value of $1.3 \times 10^{-5} \mathrm{~s}^{-1}$ at $r_{h}=1 \mathrm{AU}$ (Crovisier 1994).

To calculate the excitation and line emission, the gas temperature is also required. Lacking in situ measurements, we adopt the same temperature distribution as Figure 1 of Crovisier (1987), which the authors note is approximate at best. This profile is similar to the one adopted by Friedel et al. (2005), which was based on models developed to explain optical line profiles observed toward Hyakutake by Combi et al. (1999). Both profiles agree with the in situ measurements at $1 \mathrm{P} /$ Halley by the Giotto spacecraft Ip \& Axford (1990). To obtain an estimate as to how sensitive the calculations are to the gas temperature profile, we repeated the calculations for a constant kinetic temperature of $40 \mathrm{~K}$ (compared to Bensch \& Bergin 2004).

For the ill-constrained electron density and temperature, we follow Biver (1997) (see also Bensch \& Bergin 2004). The electron temperature $T_{e}$ is described by an inner region where collisions couple $T_{e}$ to the gas kinetic temperature $T_{\mathrm{kin}}$, an outer region where interactions with charged particles from the Solar wind determine $T_{e}$, and a transitional region in between,

$$
T_{e}= \begin{cases}T_{\mathrm{kin}}, & \text { for } r<R_{\mathrm{cs}}, \\ T_{\mathrm{kin}}+\left(10^{4} \mathrm{~K}-T_{\mathrm{kin}}\right)\left(\frac{r}{R_{\mathrm{cs}}}\right), & \text { for } R_{\mathrm{cs}}<r<2 R_{\mathrm{cs}} \\ 10^{4} \mathrm{~K}, & \text { for } r>2 R_{\mathrm{cs}},\end{cases}
$$

where $R_{\mathrm{cs}}=1125 x_{r_{e}} Q_{29}^{3 / 4} \mathrm{~km}$ is the radial location of the contact surface; $Q_{29}$ is the water production rate in units of $10^{29} \mathrm{~s}^{-1}$ and a scaling factor $x_{r_{e}} \approx 1$ is adopted. 

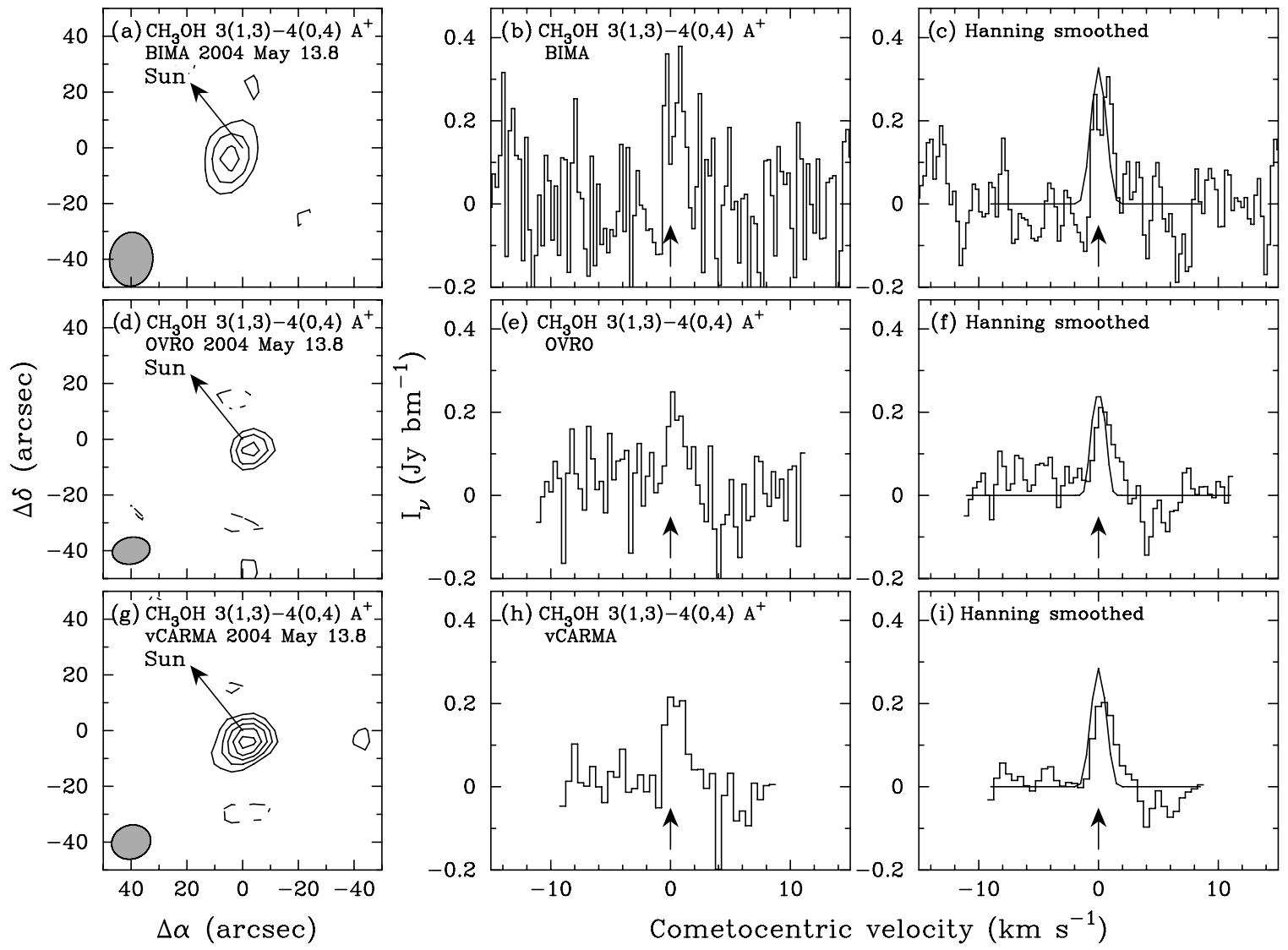

Figure 2. As Figure 1, for the $\mathrm{CH}_{3} \mathrm{OH} J\left(K_{a}, K_{c}\right)=3(1,3)-4(0,4) \mathrm{A}^{+}$line observed on 2004 May 11.
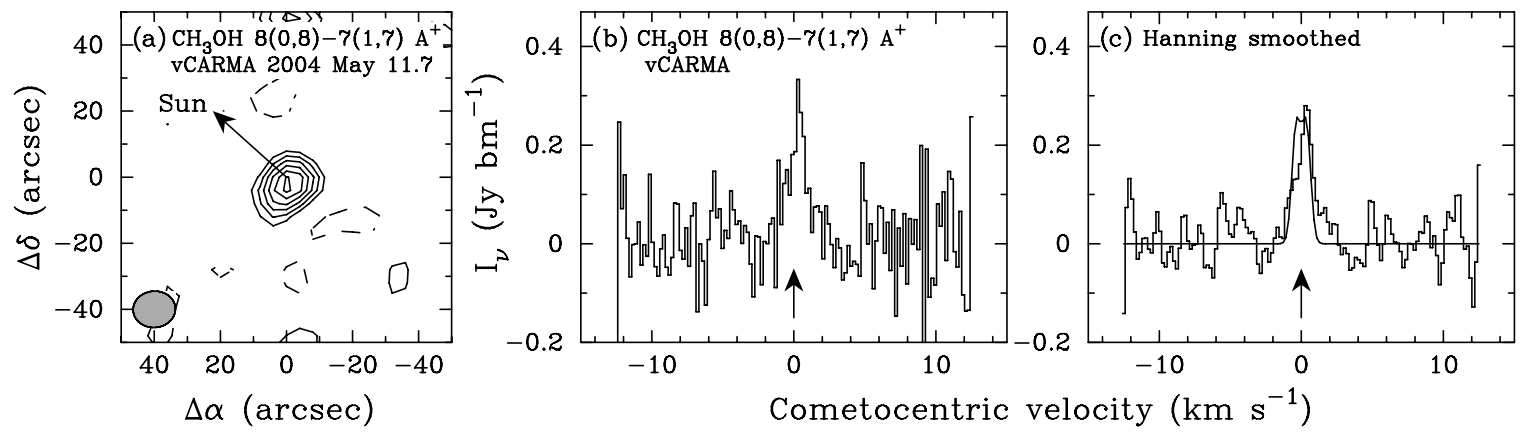

Figure 3. As Figure 2, for $\mathrm{CH}_{3} \mathrm{OH} J\left(K_{a}, K_{c}\right)=8(0,8)-7(1,7) \mathrm{A}^{+}$which was only observed with OVRO on 2004 May 13.

Assuming equilibrium between ionization and recombination, the electron density becomes

$$
\begin{aligned}
n_{e}= & x_{n_{e}}\left(\frac{Q_{\mathrm{H}_{2} \mathrm{O}} k_{\mathrm{ion}} / r_{h}^{2}}{v_{0} k_{\mathrm{rec}}}\right)^{1 / 2}\left(\frac{T_{e}}{300 \mathrm{~K}}\right)^{0.15}\left(\frac{R_{\mathrm{rec}}}{r^{2}}\right) \\
& \times\left[1-\exp \left(-\frac{r}{R_{\mathrm{rec}}}\right)\right],
\end{aligned}
$$

where the radius of the recombination surface $R_{\mathrm{rec}}=$ $3200 x_{r_{e}} Q_{29}^{1 / 2} \mathrm{~km}$ and the scaling factor $x_{n_{e}}$ is again chosen to be 1.0. The photoionization rate at $r_{h}=1 \mathrm{AU}, k_{\text {ion }}=4.1 \times$ $10^{-7} \mathrm{~s}^{-1}$ for solar minimum and $10.5 \times 10^{-7} \mathrm{~s}^{-1}$ for solar maximum (we adopt $4.1 \times 10^{-7} \mathrm{~s}^{-1}$ ). The recombination rate is given by $k_{\text {rec }}=0.7 \times 10^{-6}\left(300 \mathrm{~K} / T_{e}\right)^{1 / 2} \mathrm{~cm}^{3} \mathrm{~s}^{-1}$. The expansion velocity $v_{0}$ was chosen as $0.62 \mathrm{~km} \mathrm{~s}^{-1}$ as before. We will estimate the effect of excitation by electrons by also considering models without this term (e.g., $n_{e}=0$ ).
Figures 4 and 5 graphically illustrate some of the characteristics of the adopted comet model.

\subsection{Molecular Excitation}

We calculate the line formation and molecular excitation in the coma using an adapted version of the Accelerated Monte Carlo code by Hogerheijde \& van der Tak (2000). This includes collisional excitation of the rotational levels by water and electrons, spontaneous and induced emission of line photons, and absorption of line and continuum photons. The radial distribution of molecular emissions is modeled via shells at distances from 10 up to $10^{5} \mathrm{~km}$ from the nucleus. Each shell has constant physical properties, which vary from shell to shell. The Haser model (Equation (1)) provides the radial density distribution for the molecular $\left(\mathrm{H}_{2} \mathrm{O}, \mathrm{HCN}, \mathrm{CH}_{3} \mathrm{OH}\right)$ species. The line excitation for each shell is calculated including 


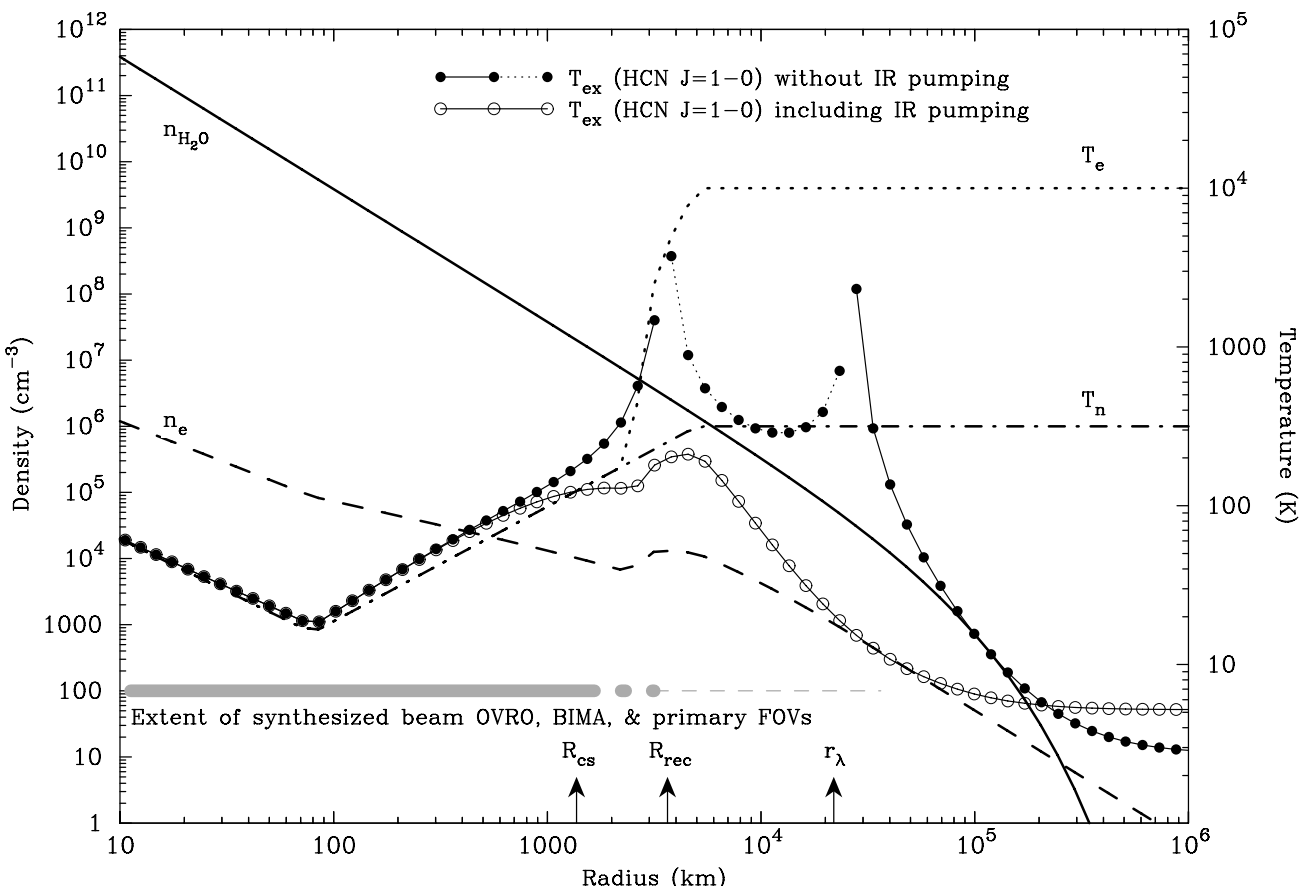

Figure 4. Adopted comet model and resulting excitation temperature of the HCN $J=1-0$ transition. The heavy lines show the number density of water (solid line) and electrons (dashed line), and the temperature of the neutral gas (dash-dotted line) and electrons (dotted line). The thin lines with symbols show the HCN $J=1-0$ excitation temperature, without infrared pumping (filled symbols) and including infrared pumping by solar photons (open symbols). Symbols connected by dotted lines indicate regions where the populations are inverted and $T_{\mathrm{ex}}<0$. Three characteristic radii of the comet model are indicated by vertical arrows: the locations of the contact surface $R_{\mathrm{cs}}$ and the recombination surface $R_{\mathrm{rec}}$ that define the electron density, and the photodissociation radius $r_{\lambda}$ that defines the extent of the water distribution. The horizontal gray lines indicate the size of the synthesized beams of OVRO (heavy solid line) and BIMA (heavy dotted line), and of the primary field of view of the arrays (thin dashed line).

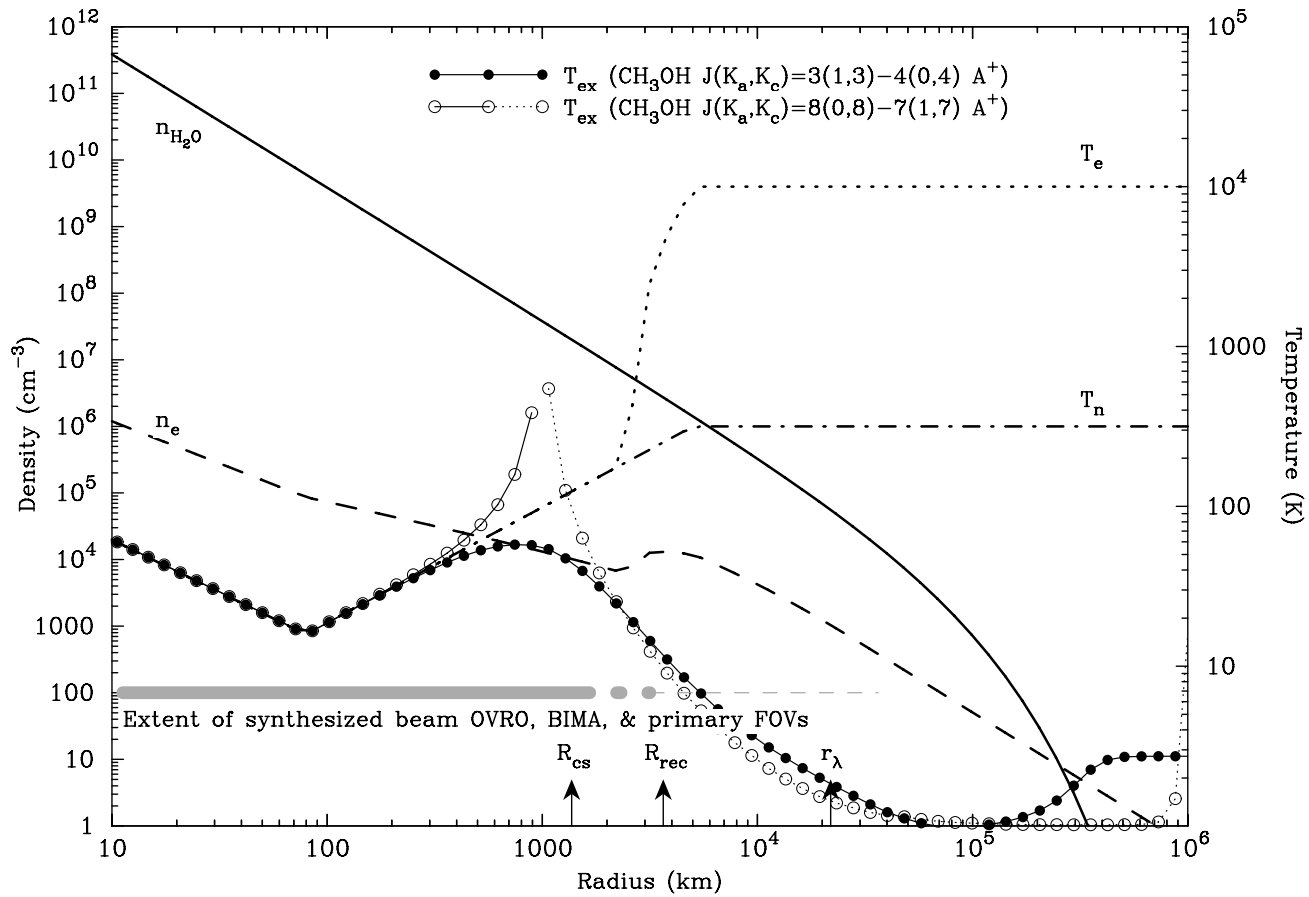

Figure 5. Adopted comet model and resulting excitation temperature of the $\mathrm{CH}_{3} \mathrm{OH} J\left(K_{a}, K_{c}\right)=3(1,3)-4(0,4) \mathrm{A}^{+}$and $8(0,8)-7(1,7) \mathrm{A}^{+}$transitions. The thin lines with symbols show the $\mathrm{CH}_{3} \mathrm{OH}$ excitation temperatures for the $3(1,3)-4(0,4) \mathrm{A}^{+}$(filled symbols) and $8(0,8)-7(1,7) \mathrm{A}^{+}$(open symbols) transitions. Otherwise, the meaning of the symbols and lines are as in Figure 4.

collisions, and radiative (de-)excitation through line photons and photons from the Cosmic Microwave Background.

We adapted the original code from Hogerheijde \& van der Tak (2000) to describe the excitation through solar infrared photons via an effective "pumping rate," following Bensch \& Bergin (2004). This method assumes that excitation from a rotational level in the vibrational ground state into the first vibrationally excited state can only happen after absorption of a solar infrared 
photon of the required frequency; collisional excitation into the vibrationally excited levels is neglected. It also assumes that each excitation event is immediately followed by radiative spontaneous de-excitation back into the ground state; collisional de-excitation is neglected, as are transitions between rotational levels within the first vibrationally excited state and excitation into higher vibrational levels. These assumptions are valid in the low density and low radiation environment of a cometary coma at $1 \mathrm{AU}$ from the Sun. The rotational selection rules for rovibrational transitions $\left(J \rightarrow J^{\prime}=\Delta J=0, \pm 1\right)$ result in effective transition rates with $J \rightarrow J^{\prime} \rightarrow J^{\prime \prime}=0, \pm 1$, and \pm 2 for excitation followed by de-excitation, each with their respective probabilities. A more detailed description of this method can be found in Bensch \& Bergin (2004).

The advantage of this approach lies in the fact that given the intensity of the solar radiation field, the effective $J \rightarrow J^{\prime \prime}$ transition rates can be easily calculated and added to the pure-rotational rates due to collisions and radiation already calculated in the code of Hogerheijde \& van der Tak (2000). No energy levels in the first vibrationally excited level need to be explicitly included in the calculation. Also, the capability of the original code to handle multidimensional geometries is maintained. While we will not use that capability in this paper to model the spherically symmetric emission, future observations of cometary emission with, e.g., the SMA, CARMA, and ALMA may profit from this approach (compared to evidence of jets in observations of Hale-Bopp; Blake et al. 1999; Woodney et al. 2002).

Since no $\mathrm{HCN}-\mathrm{H}_{2} \mathrm{O}$ collision rates are available in the literature, we modify the $\mathrm{HCN}-\mathrm{He}$ rates from Green \& Thaddeus (1974) as published online in the LAMBDA database ${ }^{15}$ and described by Schöier et al. (2005). We scale all collision rates up by a factor of 7.5 corresponding to the larger molecular weight of water, and investigate the effect on the obtained results by varying the scaling factor. Frequencies and Einstein-A coefficients of the transitions between the vibrational ground state and the first vibrationally excited state are taken from the HITRAN database. ${ }^{16}$ The hyperfine splitting of the HCN $J=1-0$ line is included by distributing the population of the molecule over the three lines in the equilibrium ratio of 1:0.2:0.6. Our calculations therefore treat the non-LTE excitation of the HCN rotational level, but adopt an LTE distribution over the hyperfine levels within the rotational level. Optical depth effects, however, will still affect the relative strengths of the HCN hyperfine lines. Since the underlying populations are kept in their equilibrium value, a detailed quantitative comparison of the relative strengths of the three lines is not possible. The solar spectrum is approximated by a $5770 \mathrm{~K}$ blackbody. For $\mathrm{CH}_{3} \mathrm{OH}$ we use collision rates from Lees (1973) and M. Walmsley (1999, private communication), scaled by the same factor of 7.5.

Our excitation calculation was validated by reproducing the pioneering calculations of molecular excitation in comets by Crovisier (1987). Using the same parameters for the comet but our own $\mathrm{HCN}-\mathrm{H}_{2} \mathrm{O}$ rates, we find $\mathrm{HCN}$ level populations that are comparable (but not identical) to those presented by Crovisier (1987), which range from an LTE region close to the nucleus to a region in fluorescent equilibrium in the outer coma. Most importantly, the transition region is reproduced accurately, where the excitation is neither dominated by collisions or by solar irradiation, but instead follows a detailed balance

\footnotetext{
15 www.strw.leidenuniv.nl/ $\sim$ moldata

16 cfa-www.harvard.edu/hitran
}

Table 3

Production Rates

\begin{tabular}{lccccc}
\hline \hline Species & $\log \left(Q / \mathrm{s}^{-1}\right)^{\mathrm{a}}$ & $\begin{array}{c}\text { Model } I_{0} \\
\left(\mathrm{Jy} \mathrm{beam}^{-1} \mathrm{~km} \mathrm{~s}^{-1}\right)\end{array}$ & $\begin{array}{c}\text { Statistical } \\
\text { Error }\end{array}$ & $\begin{array}{c}\text { Comet } \\
\text { Model } \\
\text { Accuracy }\end{array}$ & $\begin{array}{c}\text { Excitation } \\
\text { Accuracy }\end{array}$ \\
\hline $\mathrm{HCN}$ & 26.45 & $0.42^{\mathrm{b}}$ & 0.05 & 0.18 & 0.28 \\
$\mathrm{CH}_{3} \mathrm{OH}$ & 27.34 & 0.44 & 0.06 & 0.18 & 0.20 \\
\hline
\end{tabular}

Notes.

${ }^{a}$ Derived assuming a water production rate of $\log \left(Q / \mathrm{s}^{-1}\right)=29.48$; see Section 4.

${ }^{\mathrm{b}}$ Integrated intensity for the main $\mathrm{HCN} J=1-0$ hyperfine component $F=2-1$ only.

between the different processes. For Earth-comet distances of the order of $1 \mathrm{AU}$, beams of $10^{\prime \prime}-20^{\prime \prime}$ are dominated by material in LTE, even though the beam also traverses the outer regions in fluorescent equilibrium. Calculations of the integrated intensity in models with and without solar infrared pumping yield identical results, confirming the arguments of Friedel et al. (2005). Even though we expect infrared pumping to be of little effect on our results, we include the process for completeness in our HCN calculations but ignore the effect for the much more complex energy-level structure of the $\mathrm{CH}_{3} \mathrm{OH}$ molecule (compared to Bockelée-Morvan et al. 1994).

As a final step, to directly compare the model results to the observed emission, we integrate along many lines of sight through the shells making up the cometary coma model. After tapering with the appropriate primary beams, the resulting image cube is processed into two interferometric data sets using the MIRIAD task UVMODEL, using the actual BIMA and OVRO antenna positions to generate the visibilities. This modeling step explicitly takes care of the filtering properties of the respective arrays. These model visibilities, derived from the cometary model, are combined in the same way as the original data, and can be deconvolved using the same methods. The entire process was repeated (excitation; line-of-sight integrations; visibility generation; combination) for different values of the production rate $Q$ until the observed integrated intensities were reproduced accurately.

\subsection{Molecular Production Rates}

Using the comet model described in Section 4.1 and the excitation calculation described in Section 4.2, production rates of $\mathrm{HCN}$ and $\mathrm{CH}_{3} \mathrm{OH}$ are derived and listed in Table 3. We obtain our fit by matching the observed and modeled integrated intensities of the combined (vCARMA) data set; these models also reproduce the individual BIMA and OVRO integrated intensities and the spectral line shapes: Figures 1-3 compare the observations to the spectral line profiles of the models that best reproduce the observed integrated intensity. For HCN, we find a production rate of $2.9 \times 10^{26} \mathrm{~s}^{-1}$; for $\mathrm{CH}_{3} \mathrm{OH}$, we find $2.2 \times 10^{27} \mathrm{~s}^{-1}$. When taking into account the uncertainties in these numbers (discussed below), they are found to agree well with earlier results by Friedel et al. (2005) who find $Q(\mathrm{HCN})=6.5 \pm 2.2 \times 10^{26} \mathrm{~s}^{-1}$, and Remijan et al. (2008) who find $Q\left(\mathrm{CH}_{3} \mathrm{OH}\right)=2.0 \times 10^{27} \mathrm{~s}^{-1}$ (note that the latter correct an earlier estimate of $Q\left(\mathrm{CH}_{3} \mathrm{OH}\right)$ from Remijan et al. 2006 by using a better estimate of the $\mathrm{CH}_{3} \mathrm{OH}$ excitation obtained from a multiline analysis).

Table 3 also lists the accuracy of the derived production rates. We consider three sources of error: one statistical term, due to the noise of the observations ( $1 \sigma \mathrm{rms}$ noise value on the integrated intensities used for the model fit); and two systematic terms, one 
due to uncertainties in the comet model (water production rate, kinetic temperature, electron fraction, and temperature), and one due to uncertainties in the excitation calculations (treatment of infrared pumping, adopted values of the collision rates). Because of the nature of the effect of these error terms on the results, we find it most useful to discuss (and present in the table) the logarithm of the production rates and their uncertainties.

For $\mathrm{HCN}, \log (Q)=26.46$ where $Q$ is the production rate in units $\mathrm{s}^{-1}$. The statistical error due to noise corresponds to 0.05 in $\log (Q)$. The uncertainty due to the comet model amounts to 0.18 in $\log (Q)$, derived by varying the kinetic temperature (i.e., set to a constant $40 \mathrm{~K}$ ) and by ignoring electron collisions. The uncertainty due to potential errors in the excitation calculation amounts to 0.28 in $\log (Q)$, derived by scaling the collision rates by factors $0.1-10$ and by excluding infrared pumping. The latter has a negligible effect.

For $\mathrm{CH}_{3} \mathrm{OH}, \log (Q)=27.34$, with a statistical error of 0.06 in $\log (Q)$, a systematic uncertainty due to the comet model of 0.18 in $\log (Q)$, and a systematic uncertainty due to the excitation treatment of 0.20 in $\log (Q)$. For both species we find that the uncertainty is dominated in equal parts by the uncertainties in the comet model and the excitation treatment by factors 1.5-1.9 each, with a combined effect that can be as large as a factor of 3 .

The derived production rates can be converted to molecular abundances with respect to water, using the adopted $Q\left(\mathrm{H}_{2} \mathrm{O}\right)$ of $3.0 \times 10^{29} \mathrm{~s}^{-1}$ (Biver et al. 2002). We find a value for $\mathrm{HCN} / \mathrm{H}_{2} \mathrm{O}$ of $0.1 \%$ and for $\mathrm{CH}_{3} \mathrm{OH} / \mathrm{H}_{2} \mathrm{O}$ of $0.7 \%$. Both values have an uncertainty of factors $1.5-3$ as discussed above. Using a uniform analysis, Biver et al. (2002) find that $\mathrm{HCN} / \mathrm{H}_{2} \mathrm{O}$ is nearly identical for many comets at $0.1 \%$, and comet LINEAR is no exception if we take our result at face value. Our $\mathrm{CH}_{3} \mathrm{OH} /$ $\mathrm{H}_{2} \mathrm{O}$ of $0.7 \%$ is on the low end of the range quoted by BockeléeMorvan et al. (2004) of $<0.15 \%$ to $6 \%$, and lower than the $\sim 2 \%$ that appears the most common value (Biver et al. 2006). The estimated accuracy of our derived productions rates of a factor 1.5-3 is comparable to the range of $\mathrm{HCN} / \mathrm{H}_{2} \mathrm{O}$ ratios quoted by Biver et al. (2002), suggesting that perhaps the intrinsic $\mathrm{HCN}$ abundance has an even narrower range. The $\mathrm{CH}_{3} \mathrm{OH} / \mathrm{H}_{2} \mathrm{O}$ ratios presented by Biver et al. (2002) and Bockelée-Morvan et al. (2004) vary by as much as a factor of 7, reflecting a real abundance variation. Even though Biver et al. (2002) derive abundances using the same method, we stress that the size of the thermalized region depends on the adopted collision rates and on the water production rate (and, to a minor extent, the electron fraction and collision rates). This means that within a similar sized beam (e.g., $\sim 15^{\prime \prime}$ ), comets with a higher water production rate $\left(\gtrsim 10^{29} \mathrm{~s}^{-1}\right)$ are better approximated by LTE excitation conditions than comets with lower production rates $\left(\lesssim 10^{28} \mathrm{~s}^{-1}\right)$. Consequently, model uncertainties will have a larger effect on the derived abundances in the latter cases compared to the former ones. For example, in the case of comet LINEAR, increasing the collision rates by a factor of 10 (which is equivalent to increasing the production rate and therefore number density of water by the same factor), decreases the derived production rates of $\mathrm{HCN}$ and $\mathrm{CH}_{3} \mathrm{OH}$ only by $10 \%-20 \%$. On the other hand, decreasing the collision rates by a factor of 10 , increases the derived $Q$ by $50 \%-100 \%$. This asymmetric behavior occurs because for lower collision rates a larger fraction of the beam samples subthermally excited gas; for a higher collision rate, the beam is already mostly filled with gas in thermodynamic equilibrium, and the emission will only depend on the temperature but no longer on the efficiency of the collisions. Figures 4 and 5 illustrate that the synthesized beams of our BIMA, OVRO (and, by extension, vCARMA) observations effectively trace material in LTE for HCN. The excitation temperatures of the observed transitions lie close to the kinetic temperature on the size scale of the beam, but diverge outside this region. For $\mathrm{CH}_{3} \mathrm{OH}$, non-LTE material partially fills the beams in all cases.

We therefore conclude that especially methods that assume LTE, but more generally any method that has some uncertainty in collision rates or cometary structure, will be less accurate for comets with a low water production rate compared to comets with a higher water production rate. The boundary between "low" and "high" production rates depends on the beam size and the critical density of the observed transitions $\left(n\left(\mathrm{H}_{2} \mathrm{O}\right)_{\text {crit }}=4 \times 10^{5}\right.$ for $\mathrm{HCN} 1-0$ and $4-5 \times 10^{4} \mathrm{~cm}^{-3}$ for both $\mathrm{CH}_{3} \mathrm{OH}$ lines, using the adopted collision rates); for the data presented here, the cutoff lies around $10^{28} \mathrm{~s}^{-1}$. The smaller beams offered by millimeter interferometers are therefore also to be preferred over the larger single-dish beams, which are more prone to include material out of LTE.

A robust way to overcome the excitation problem is mentioned by Bockelée-Morvan et al. (2004) and recently illustrated by Remijan et al. (2008): using multiple transitions, the molecular excitation can be observationally constrained; if only a single line is observed, the excitation has to be assumed (e.g., LTE) or calculated using a specific model like the one presented in Sections 4.1 and 4.2. If, on the other hand, the molecular excitation is observationally constrained from multiple observed transitions, one can derive the column densities and production rates of molecules without resorting to assumptions. The $\mathrm{CH}_{3} \mathrm{OH}$ excitation temperature and production rate derived by Remijan et al. (2008) agree well with our reported value, validating the assumptions that went into our comet model and excitation treatment. The increasing capabilities for multiline observations of existing interferometers (SMA, CARMA, or the IRAM Plateau de Bure interferometer) and the many receiver bands of the future Atacama Large Millimeter/Submillimeter Array (ALMA) offer the possibility to obtain model-independent constraints on the molecular abundances in cometary comae.

\section{SUMMARY}

We present observations of $\mathrm{HCN} J=1-0$ and $\mathrm{CH}_{3} \mathrm{OH}$ $J\left(K_{a}, K_{c}\right)=3(1,3)-4(0,4) \mathrm{A}^{+}$emission from comet C/2002 T7 (LINEAR) obtained simultaneously with BIMA and OVRO, and of $\mathrm{CH}_{3} \mathrm{OH} J\left(K_{a}, K_{c}\right)=8(0,8)-7(1,7) \mathrm{A}^{+}$obtained with OVRO. All three lines are clearly detected, and we derive production rates of $\mathrm{HCN}$ and $\mathrm{CH}_{3} \mathrm{OH}$ using an excitation method that takes into account collisions with water and electrons, and absorption of (line and continuum) photons. For $\mathrm{HCN}$, we explicitly take into account pumping of the rotational levels through infrared excitation by solar photons followed by spontaneous de-excitation. Our main conclusions are as follows.

1. We derive a production rate for $\mathrm{HCN} Q$ in units $\mathrm{s}^{-1}$ of $\log (Q)=26.45 \pm 0.05 \pm 0.18 \pm 0.28$ and for $\mathrm{CH}_{3} \mathrm{OH}$ of $\log (Q)=27.34 \pm 0.06 \pm 0.18 \pm 0.20$. Here the first error estimate corresponds to the noise, the second to the uncertainties in the adopted comet model, and the third to the uncertainties in excitation treatment. In percentages, these error contributions correspond to, respectively, $\sim 10 \%, \sim 50 \%$, and $\sim 100 \%$ (or a factor of 2 ).

2. Our derived production rates correspond to abundances of $\mathrm{HCN} / \mathrm{H}_{2} \mathrm{O}$ of $\sim 0.1 \%$ and $\mathrm{CH}_{3} \mathrm{OH} / \mathrm{H}_{2} \mathrm{O}$ of $\sim 0.7 \%$. However, the systematic uncertainties due to the comet 
model and molecular excitation are large, amounting to factors of $1.5-3$. The obtained $\mathrm{HCN} / \mathrm{H}_{2} \mathrm{O}$ ratio is consistent with the value found toward other comets (BockeléeMorvan et al. 2004), while the $\mathrm{CH}_{3} \mathrm{OH} / \mathrm{H}_{2} \mathrm{O}$ ratio is on the low end of the commonly inferred range. In that respect, comet LINEAR may be similar to comet C/1999 $\mathrm{S} 4$ (LINEAR) with an inferred $\mathrm{CH}_{3} \mathrm{OH} / \mathrm{H}_{2} \mathrm{O}$ of $\leqslant 0.96 \%$ (Bockelée-Morvan et al. 2001).

3. The systematic uncertainties can be significant relative to reported variations of production rates between comets. They therefore affect the ability to compare the molecular abundances between different dynamic comet families. Even when using the same method to derive production rates, its accuracy depends critically on such parameters as the water production rate and the resulting size of the LTE region.

4. Multiline studies that directly constrain the molecular excitation should provide much more reliable abundance estimates. Comparison of our $\mathrm{CH}_{3} \mathrm{OH}$ value to one obtained from such a study for the same comet, Remijan et al. (2008) show that, at least for this species, our adopted comet model and excitation treatment is accurate.

We are indebted to the staffs of BIMA and OVRO for making the observations possible. Frank Bensch is thanked for useful discussions about the required adaptations of the molecular excitation code. Fred Lahuis is thanked for help with the vibrational spectroscopy. The Netherlands Research School for Astronomy (NOVA) is acknowledged for a bezoekersbeurs for Imke de Pater to Leiden. The anonymous referee is thanked for many useful comments that greatly improved the manuscript.

\section{REFERENCES}

Bensch, F., \& Bergin, E. A. 2004, ApJ, 615, 531

Biver, N. 1997, PhD thesis, Université de Paris VII-Diderot
Biver, N., et al. 2002, Earth Moon Planets, 90, 323

Biver, N., et al. 2006, A\&A, 449, 1255

Biver, N., et al. 2007, Planet. Space Sci., 55, 1058

Blake, G. A., Qi, C., Hogerheijde, M. R., Gurwell, M. A., \& Muhleman, D. O. 1999, Nature, 398, 213

Bockelée-Morvan, D., Crovisier, J., Colom, P., \& Despois, D. 1994, A\&A, 287, 647

Bockelée-Morvan, D., Crovisier, J., Mumma, M. J., \& Weaver, H. A. 2004, in Comets II, ed. M. C. Festou et al. (Tucson, AZ: Univ. of Arizona Press), 391

Bockelée-Morvan, D., et al. 2001, Science, 292, 1339

Combi, M. R., Cochran, A. L., Cochran, W. D., Lambert, D. L., \& Johns-Krull, C. M. 1999, ApJ, 512, 961

Crovisier, J. 1987, A\&AS, 68, 223

Crovisier, J. 1994, J. Geophys. Res., 99, 3777

Crovisier, J. 2007, arXiv:astro-ph/0703785

DiSanti, M. A., Bonev, B. P., Magee-Sauer, K., Russo, N. D., Mumma, M. J., Reuter, D. C., \& Villanueva, G. L. 2006, ApJ, 650, 470

Friedel, D. N., et al. 2005, ApJ, 630, 623

Green, S., \& Thaddeus, P. 1974, ApJ, 191, 653

Haser, L. 1957, Bull. Soc. R. Sci. Liege, 43, 740

Hogerheijde, M. R., \& van der Tak, F. F. S. 2000, A\&A, 362, 697

Hogerheijde, M. R., et al. 2004, AJ, 127, 2406

Ip, W. H., \& Axford, W. I. 1990, in Physics and Chemistry of Comets, ed. F. Huebner (Berlin: Springer), 177

Lees, R. M. 1973, ApJ, 184, 763

Lovell, A. J., Schloerb, F. P., Dickens, J. E., De Vries, C. H., Senay, M. C., \& Irvine, W. M. 1998, ApJ, 497, L117

Milam, S. N., et al. 2006, ApJ, 649, 1169

Pickett, H. M., Poynter, I. R. L., Cohen, E. A., Delitsky, M. L., Pearson, J. C., \& Muller, H. S. P. 1998, J. Quant. Spectrosc. Radiat. Transfer, 60, 883

Remijan, A. J., et al. 2006, ApJ, 643, 567

Remijan, A. J., et al. 2008, ApJ, 689, 613

Sault, R. J., Teuben, P. J., \& Wright, M. C. H. 1995, in ASP Conf. Ser. 77, Astronomical Data Analysis Software and Systems IV, ed. R. E. Shaw, H. E. Payne, \& J. J. E. Hayes (San Francisco, CA: ASP), 433

Schöier, F. L., van der Tak, F. F. S., van Dishoeck, E. F., \& Black, J. H. 2005, A\&A, 432, 369

Scoville, N. Z., Carlstrom, J. E., Chandler, C. J., Phillips, J. A., Scott, S. L., Tilanus, R. P. J., \& Wang, Z. 1993, PASP, 105, 1482

Womack, M., Homich, A., Festou, M. C., Mangum, J., Uhl, W. T., \& Stern, S. A. 1997, Earth Moon Planets, 77, 259

Woodney, L. M., et al. 2002, Icarus, 157, 193

Wright, M. C. H., et al. 1998, AJ, 116, 3018 